\title{
Synthesis and Application of Oil-Soluble Red Dyes Derived from p-n-Alkyl Aniline
}

\author{
Yi-Fen Chiang, Yu-Chou Chao \\ Institute of Organic and Polymer Materials, National Taipei University of Technology, Taipei, Chinese Taipei \\ Email: carol.chiang@jintex.com.tw
}

Received 14 March 2014; revised 24 April 2014; accepted 10 May 2014

Copyright @ 2014 by authors and Scientific Research Publishing Inc.

This work is licensed under the Creative Commons Attribution International License (CC BY). http://creativecommons.org/licenses/by/4.0/

(c) (i) Open Access

\begin{abstract}
In this study, the azo red dyes derived from p-n-alkyl aniline by the introduction of different alkyl groups having high solubility in dodecane were synthesized. Results indicated that the elementary properties of red oil inks were 1) non-polarity; 2) low viscosity ( $<3.0 \mathrm{cps}$ ); 3) specified surface tension $(<30 \mathrm{mN} / \mathrm{m}) ; 4)$ intensity of visible absorption covering $480-540 \mathrm{~nm}$; 5 ) hue close to standard red $(\mathrm{CIE}(\mathrm{x}, \mathrm{y})=\mathbf{0 . 6 7}, 0.33)$. We can conclude that these azo red dyes are applicable for electrowetting displays.
\end{abstract}

\section{Keywords}

Oil-Soluble Red Dyes, Dodecane, Electrowetting, Display

\section{Introduction}

Much research in recent years has demonstrated that electrowetting technology is an attractive technology for the rapid manipulation of liquids on a micrometer scale [1]-[4]. The reflectivity of these displays is four times that of LCDs [5] and the response speed is significantly faster than that of electrophoretic displays, making it possible to display video content [6]. Feenstra and Hays present an electrowetting-based reflective display with potential for use in the field of electronic paper [7].

Liquavista propose possible mode of color electrowetting display with multi-layer structure. The multi-layer structure is composed of blue, red, and green (three-color) oil layers [8]. This structure has higher light utilization and several additional advantages. In the electrophoresis type (E-link) electronic paper technology the reflectance $(\mathrm{R})$ value is close to $40 \%$ [9]. Electrowetting displays provide a high white state reflectance of $>50 \%$ [10]. In this technology colored ink with high optical density covers a white reflective substrate, the reflectance of the display is higher and contrast is better.

In this case colored oil ink functions as an absorbing switch. The colored oil should possess the specific sur- 
face tension and be compatible with the insulator [11]. Few commercial dyes satisfy the requirements which include neutral hue, high solubility in non-polar solvent, high molar absorption coefficient and low viscosity. Furthermore, as the concentration of dye in the oil ink increases, the oil-water interfacial tension decreases [12] and the viscosity of the oil ink increases steeply. This effect may influence either the amplitude of the driving voltage or the speed of oil movement when voltage is applied [13].

Commercial red dyes such as Sudan Red, Solvent Red 164 and Sandoplast Red BB possess poor solubility in non-polar solvents and the shade of these red dyes is not close to standard red (CIE (x, y) $=0.67,0.33)$. The purpose of this study was to synthesize a series of azo dyes derived from p-n-alkyl aniline by introducing different alkyl groups possessing high solubility in dodecane and having standard hues. The red oil inks have the advantages of a neutral red hue, specific surface tension and low viscosity.

\section{Experimental}

\subsection{Chemicals and Reagents}

All reagents used in the present study were pure grade available from Aldrich or Sigma. The solvents used were analytical grade from Merck or Aldrich.

\subsection{Equipment}

UV-Vis Spectroscopy (U-3010, Hitachi), Nuclear magnetic resonance (NMR, Bruker AV500), Fourier transform spectroscopy (FTIR-460 Plus). The properties of the oils were measured with an SV-10 Sine-wave Vibro Viscometer and a SITA pro line f10 tensiometer.

\section{Preparation of Red Dyes and Oil Inks}

The azo compound used in this study was represented by the following formula (4) named (4-alkyl-phenyl)(2,2-dialkyl-2,3-dihydro-1H-perimidin-6-yl)-diazene:

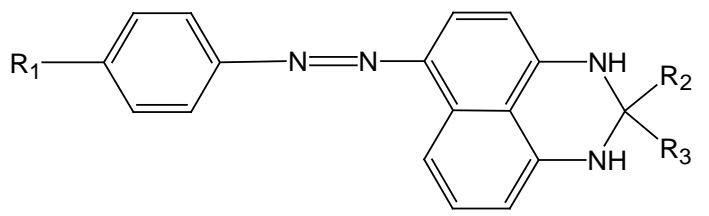

(4-alkyl-phenyl)-(2,2-dialkyl-2,3-dihydro-1H-perimidin-6-yl)-diazene

wherein $\mathrm{R}_{1}$ was substituted C4-C16 alkyl, and $\mathrm{R}_{2}$ and $\mathrm{R}_{3}$ were ethyl.

As shown in those schemes, the azo compound represented in formula (4) was synthesized by the following process. Firstly, 1,8-diaminonaphthalene was reacted with the reagent of formula (1) to obtain the intermediate of formula (2) named 2,2-dialkyl-2,3-dihydro-1H-perimidine, as shown in Scheme 1. Subsequently, the compound of formula (3) was diazotized, and then reacted with the intermediate of formula (2) to obtain the azo compound of formula (4), as shown in Scheme 2.

\subsection{Condensation of Formula (2)}

1,8-Diaminonaphthalene (1.64 g) was added to anhydrous ethanol (50 mL). The solution was stirred at room

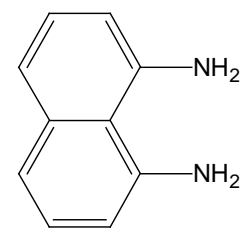

1,8-Diaminonaphthalene

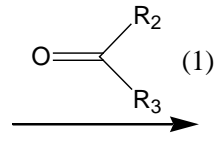

(1)

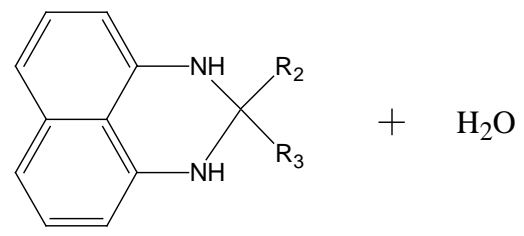

(2)

Scheme 1. Condensation reaction of formula (2). 
<smiles>Nc1ccc(Br)cc1</smiles>

(3)
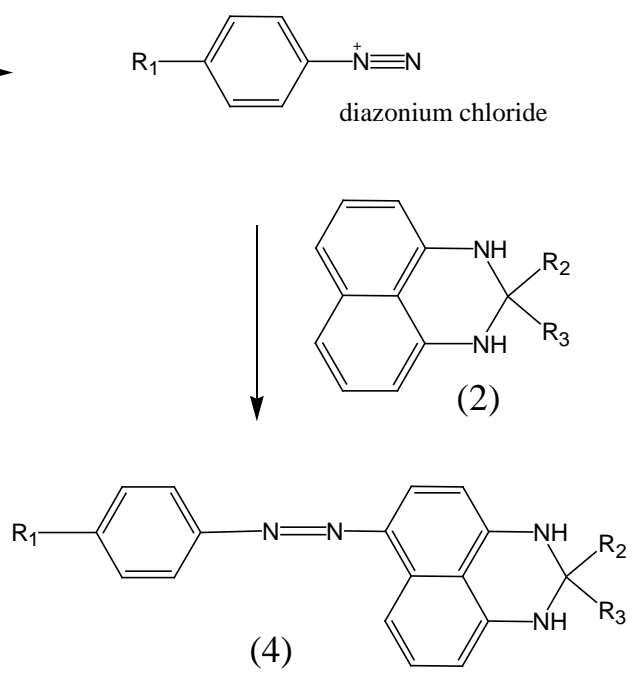

Scheme 2. Synthesis of formula (4).

temperature until uniformly dispersed. After stirring for 0.5 hour, 4-methylbenzenesulfonic acid $(0.04 \mathrm{~g})$ and 3-pentanone (1.3 g) were added and the solution was heated and kept at $60^{\circ} \mathrm{C}$. After refluxing for 24 hours, $10 \%$ ammonium solution $(50 \mathrm{~mL}$ ) was slowly added to the resulting mixture. The reaction solution was cooled to room temperature and filtered to obtain a crude wet cake of formula (2), in which $R_{2}$ and $R_{3}$ were both ethyl. The structure of the compound was identified by NMR and FTIR spectroscopy and shown to be as follows: ${ }^{1} \mathrm{H}$ NMR (CDCl $3,400 \mathrm{MHz}): \delta_{\mathrm{H}}=7.19(2 \mathrm{H}, \mathrm{e}), 7.09(2 \mathrm{H}, \mathrm{f}), 6.44(2 \mathrm{H}, \mathrm{d}), 3.73(2 \mathrm{H}, \mathrm{c}), 1.73(4 \mathrm{H}, \mathrm{b}), 0.96(6 \mathrm{H}, \mathrm{a})$. The characteristic absorptions of FTIR at $3371 \mathrm{~cm}^{-1}$ (Ar-NH-R, N-H stretching), $1376 \mathrm{~cm}^{-1}\left(\mathrm{CH}_{3}\right.$, C-H deformation vibration), $810 \mathrm{~cm}^{-1}$ (aromatic C-H out of plane).

(e) (d)

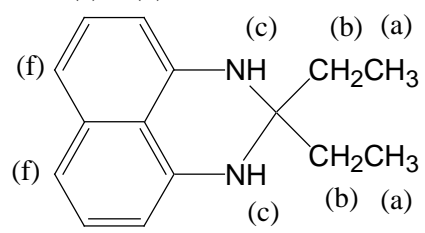

(e) (d)

2,2-Diethyl-2,3-dihydro-1H-perimidine

\subsection{Synthesis of Azo Red Dyes}

p-n-Decylaniline $(2.48 \mathrm{~g})$ was dissolved in IPA $(6 \mathrm{~mL})$ and the solution was stirred at room temperature until completely dissolved. After that $5 \mathrm{~N} \mathrm{HCl}(50 \mathrm{~mL})$ was introduced; the reaction solution was cooled to below $5^{\circ} \mathrm{C}$ in melting ice, and $40 \% \mathrm{NaNO}_{2}(7 \mathrm{~mL})$ was added and stirred for 3 hours. When the color of the solution became a clear yellow, sulfamic Acid (2.9 g) was added to remove the excess $\mathrm{HNO}_{2}$. The resulting solution was diazonium component-1.

$2.3 \mathrm{~g}$ of Formula (2) prepared above was added to methanol $(50 \mathrm{~mL})$, and stirred at room temperature for 0.5 hour. After which the reaction solution was slowly added to the diazonium component- 1 . The reaction temperature was kept under $5^{\circ} \mathrm{C}$. After 1 hour, a red compound appeared to disperse in the reaction solution, $10 \%$ ammonium solution was added to adjust the $\mathrm{pH}$ to 3 . At this time, the dyes were filtered to obtain a crude azo compound of formula (4), wherein $R_{1}$ is $n$-decyl; $R_{2}$ and $R_{3}$ were both ethyl.

The structure of the compound was identified by NMR and FTIR spectra and shown to be as follows: ${ }^{1} \mathrm{H}$ $\operatorname{NMR}\left(\mathrm{CDCl}_{3}, 400 \mathrm{MHz}\right): \delta_{\mathrm{H}}=7.91-7.86(3 \mathrm{H}, \mathrm{f}, \mathrm{e}), 7.74-7.66(2 \mathrm{H}, \mathrm{g}), 7.32-7.25(2 \mathrm{H}, \mathrm{k}, \mathrm{l}), 6.96-7.02(2 \mathrm{H}$, m, d), 3.63 - 3.62 (2H, c), 2.67 - 2.58 (2H, h), 1.99 - $1.93(4 \mathrm{H}, \mathrm{b}), 1.30$ - $1.17(16 \mathrm{H}, \mathrm{i}), 1.09$ - 0.99 (3H, j), 0.86 $0.83(6 \mathrm{H}, \mathrm{a})$. The characteristic absorptions of FTIR at $3370 \mathrm{~cm}^{-1}$ (Ar-NH-R, N-H stretching), $2925-2854 \mathrm{~cm}^{-1}$ (C-H stretching vibration), $1356 \mathrm{~cm}^{-1}\left(\mathrm{CH}_{3}, \mathrm{C}-\mathrm{H}\right.$ deformation vibration), $828 \mathrm{~cm}^{-1}$ (aromatic C-H out of plane). 
(g) (f) $\quad$ (e) (d)

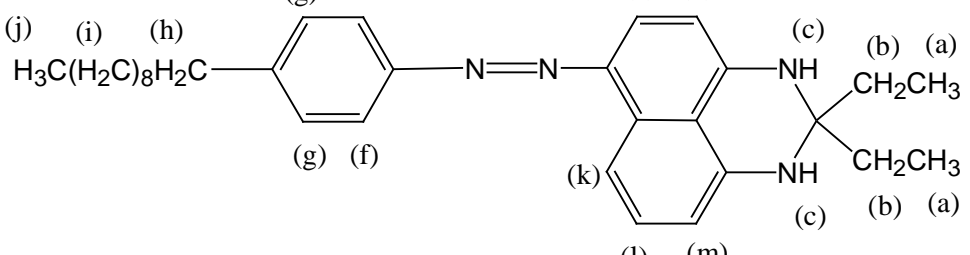

(l) (m)

(4-decyl-phenyl)-(2,2-diethyl-2,3-dihydro-1H-perimidin-6-yl)-diazene

\subsection{Synthesis of Other Embodiments}

The azo compounds of formula (4) were prepared as mentioned above. The corresponding compound of formula (4) and reagent used were listed in Table 1.

\subsection{The Preparation of Red Oil Inks}

Weighed azo red dye was dissolved in dodecane and heated to $80^{\circ} \mathrm{C}$ for one hour. The mixture was then filtered through a $0.45 \mu \mathrm{m}$ filter to obtain the oil ink. The oil ink spectrum was measured using a UV-Vis spectrophotometer and then converted to obtain the data as stimulus values of CIE chromaticity coordinates (X, Y), wherein the concentration of oil ink was $3.8 \times 10^{-5} \mathrm{M}$. The tested results were shown in Figure 1, Table 2 and Table 3 .

\subsection{Performance Test Device for the Electrowetting Red Oil Inks}

The electrowetting test device was a $5 \times 5 \mathrm{~cm}^{2}$ ITO coated glass sheet with a water-resistant paste seal, that had layers arranged in the following order: a white substrate, a hydrophobic (polyimide, PI) dielectric layer with a thickness of $0.8 \mu \mathrm{m}$, a red oil ink layer with a thickness of $7.5 \mu \mathrm{m}$, pixel size was $302 \times 302 \mu \mathrm{m}^{2}$, the thickness of the water layer was $200 \mu \mathrm{m}$.

\section{Results}

The oil inks were obtained by dissolving the azo red dyes in dodecane. Table 2 and Table 3 showed the maximum absorption wavelength, molar absorption coefficient, and the maximum solubility of the azo red dyes. The results indicated DA-C2,2 showing the highest maximum solubility where the concentration of oil ink was 23.94\% (w/w). As shown in Figure 1, the maximum absorption wavelength of visible absorption from the UVVIS spectra of DA-C2,2 was $492 \mathrm{~nm}$. As can be seen in Table 3, the shade of DA-C2,2 (CIE(x, y) = 0.64, 0.30) was closer to standard red $(\operatorname{CIE}(x, y)=0.67,0.33)$ than conventional Sudan Red $(\operatorname{CIE}(x, y)=0.51,0.33)$. The viscosity and surface tension were measured and the concentration of the oil ink was $13 \%(\mathrm{w} / \mathrm{w})$ except BA$\mathrm{C} 2,2$.

Electrowetting elements containing colored oil and water were able to be used as pixel engines for videospeed reflective display. As shown in Figure 2(a), a red oil homogeneous film was formed by DA-C2,2 at a concentration of $13 \%(\mathrm{w} / \mathrm{w})$ when no voltage was applied, and Figure 2(b) the oil film contracted dramatically when a voltage $(-15 \mathrm{~V})$ was applied. When the oil contracted, the white area fraction WA\% (the pixel area without oil) was close to $80 \%$.

\section{Discussion}

In this study, we chose dodecane as the solvent because its specific properties, such as viscosity $(1.34 \mathrm{cP}$ at $\left.25^{\circ} \mathrm{C}\right)$, vapor pressure $\left(0.3 \mathrm{mmHg}\right.$ at $\left.20^{\circ} \mathrm{C}\right)$ and surface tension $\left(25.35 \mathrm{mN} / \mathrm{m}\right.$ at $\left.20^{\circ} \mathrm{C}\right)$ were able to meet the requirements for the electrowetting display [13]. In Table 2 indicated when the alkyl group of azo component was changed, the maximum absorption wavelength and full width at half maximum of all of the red dyes were close to each other. It meant that a series of azo dyes derived from p-n-alkyl aniline by introducing different alkyl groups showing the same hue.

In Table 3, as the alkyl group of azo component became longer (R1 = n-butyl to n-decyl ) and R2, R3 were ethyl groups of coupling components, the maximum solubility of the dye increased. However, when the alkyl group of azo component became longer than DA-C2,2 such as when R1 was n-dodecyl, n-hexadecyl, the maxi- 
Table 1. The corresponding code name of dyes made and reagents (IV) used.

\begin{tabular}{ccc}
\hline Code name of dyes & $\mathrm{R}_{1}$ & Reagents (IV) used \\
\hline BA-C2,2 & n-butyl & 4-butylaniline \\
OA-C2,2 & n-octyl & 4-octylaniline \\
DA-C2,2 & n-decyl & 4-decylaniline \\
DDA-C2,2 & n-dodecyl & 4-dodecylaniline \\
HDA-C2,2 & n-hexadecyl & 4-hexadecylaniline \\
\hline
\end{tabular}

Table 2. Comparison of absorption properties of the dyes dissolved in dodecane.

\begin{tabular}{|c|c|c|c|}
\hline Code name of dye & $\begin{array}{c}\lambda_{\max } \\
\text { Absorption wavelength }(\mathrm{nm})\end{array}$ & $\begin{array}{c}\varepsilon_{\max } \\
\text { Molar absorption coefficient }\end{array}$ & $\begin{array}{l}\qquad v_{1 / 2}(\mathrm{~nm}) \\
\text { Full width at half maximum }\end{array}$ \\
\hline $\mathrm{BA}-\mathrm{C} 2,2$ & 491 & $4.59 \times 10^{3}$ & 115 \\
\hline $\mathrm{OA}-\mathrm{C} 2,2$ & 495 & $1.14 \times 10^{4}$ & 115 \\
\hline DA-C2,2 & 492 & $1.71 \times 10^{4}$ & 113 \\
\hline DDA-C2,2 & 491 & $1.31 \times 10^{4}$ & 120 \\
\hline HDA-C2,2 & 491 & $1.13 \times 10^{4}$ & 120 \\
\hline
\end{tabular}

Full width at half maximum is defined as wavelength range of half height of absorption intensity at maximum absorption wavelength $\left(\lambda_{\max }\right)$. The shade will be duller as the data becomes bigger.

Table 3. The physical properties of the dyes dissolved in dodecane.

\begin{tabular}{ccccc}
\hline Code name of dye & Maximum solubility (\%) & Viscosity (cps) & Surface tension (mN/m) & -- \\
\hline BA-C2,2 & 2.03 & -- & 24.4 & -- \\
OA-C2,2 & 13.00 & 2.65 & 24.6 & $(0.64,0.30)$ \\
DA-C2,2 & 23.94 & 2.75 & 24.3 & -- \\
DDA-C2,2 & 17.10 & 2.78 & 24.8 \\
HDA-C2,2 & 16.77 & 2.90 & -- \\
\hline
\end{tabular}

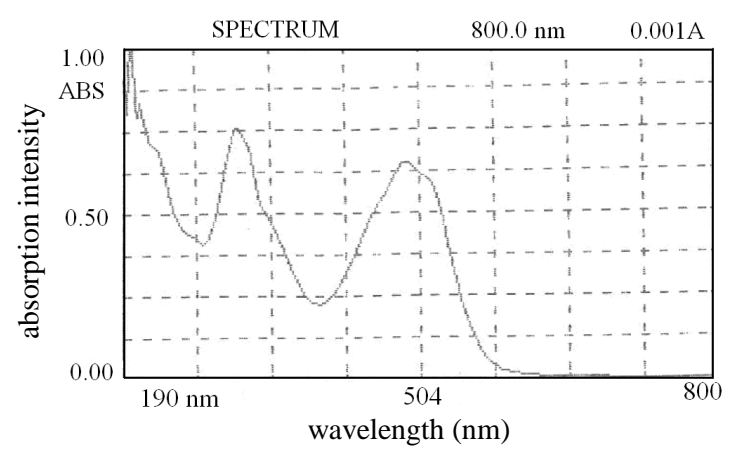

Figure 1. Shows UV-Vis absorption spectra of the oil ink (DA-C2,2). The maximum absorption wavelength of visible absorption from the UV-VIS spectra was $492 \mathrm{~nm}$.

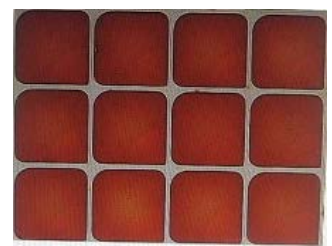

(a) Without Voltage

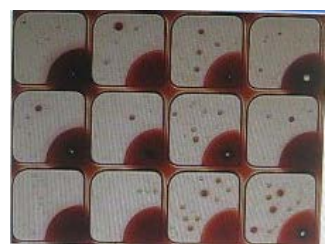

(b) With Voltage

Figure 2. Electrowetting-based optical device with a moving oil/water interface. Top view images of the oil contraction in pixels when viewed through microscope (a) off-state, no voltage applied $\mathrm{V}=0$ and (b) on-state showing oil contraction to a corner, with voltage applied, $\mathrm{V}=-15$. 
mum solubility of the dye decreased. The possible reason for this should be long carbon chain winding.

These results indicated the maximum solubility of dyes dissolved in dodecane was clearly affected by the different alkyl groups on the coupling component.

\section{Conclusion}

The study describes azo red dyes that have the advantages of standard red hue and high solubility in dodecane. We can conclude that DA-C2,2 fulfils the basic requirements of a red oil ink for electrowetting displays. Further, the red oil may be combination with blue and green oil that can be used in multi-layer structures to provide a full-color electrowetting-based reflective display as Liquavista has proposed. At present, the red oil ink is applicable for SiPix of electrophoretic displays (EPD) as fluid liquid.

\section{References}

[1] Roques-Carmes, T., Hayes, R.A. and Schlangen, L.J.M. (2004) Liquid Behavior inside a Reflective Display Pixel Based on Electrowetting. Journal of Applied Physics, 96, 6267-6271. http://dx.doi.org/10.1063/1.1810192

[2] Hayes, R.A., Joulaud, M., Roques-Carmes, T.J.B. and Palmier, S.A. (2005) Display Device Based on Electrowetting Effect. USP 359245.

[3] Feenstra, B.J., Hayes, R.A., van Dijk, R., Boom, R.G.H., et al. (2006) Electrowetting-Based Displays: Bringing Micro fluidics Alive On-Screen. 19th IEEE International Conference on Micro Electro Mechanical Systems, Istanbul, 2006, 48-53.

[4] Kim, D.Y. and Steckl, A.J. (2007) Liquid-State Field-Effect Transistors Using Electrowetting. Applied Physics Letters, 90, Article ID: 043507.

[5] Mugele, F. and Baret, J.-C. (2005) Electrowetting: From Basics to Application. Journal of Physics: Condensed Material, 17, R737-R740.

[6] Feenstra, B.J., et al. (2003) A Video-Speed Reflective Display Based on Electrowetting: Principle and Properties. Journal of the Society for Information Display, 12, 193-299.

[7] Hayes R.A. and Feenstra, B.J. (2003) Video-Speed Electronic Paper Based on Electrowetting. Nature, 425, 383-385. http://dx.doi.org/10.1038/nature01988

[8] Hayes, R.A., Feenstra, B.J. and Schlangen, L.J.M. (2009) Electrowetting Displays. Copyright Liquavista BV, 1-15.

[9] Comiskey, B., Albert, J.D., Yoshizawa, H. and Jacobson, J. (1998) An Electrophoretic Ink for All-Printed Reflective Electronic Displays. Nature, 394, 253-255. http://dx.doi.org/10.1038/28349

[10] Heikenfeld, J., Smith, N., Dhindsa, M., Zhou, K., Kilaru, M., Hou, L., Zhang, J., Kreit, E. and Raj, B. (2009) Recent Progress in Arrayed Electrowetting Optics. OPN, 20, 21-26.

[11] Berry, S., Kedzierski, J. and Abedian, B. (2006) Low Voltage Electrowetting for Microfluidic Devices. Journal of Colloid and Interface Science, 303, 517-524. http://dx.doi.org/10.1016/j.jcis.2006.08.004

[12] Roques-Carmes, T., Palmier, S., Hayes, R.A. and Schlangen, L.J. M. (2005) The Effect of the Oil/Water Interfacial Tension on Electrowetting Driven Fluid Motion. Colloids and Surfaces A: Physicochemical and Engineering Aspects, 267, 56-63.

[13] Chiang, Y.F. and Chao, Y.C. (2012) Synthesis of Dis-Azo Black Dyes for Electrowetting Displays. Materials Science and Engineering B, 177, 1672-1677. http://dx.doi.org/10.1016/j.mseb.2012.08.011 